E. OMACHI

KODAI MATH. J.

9 (1986), 170-174

\title{
HYPERSURFACES WITH HARMONIC CURVATURE IN A SPACE OF CONSTANT CURVATURE
}

\author{
BY ERIKO OMACHI
}

\section{Introduction and theorems}

A Riemannian curvature tensor $R$ is said to be harmonic if it satisfies

$$
\nabla_{i} R_{j k}-\nabla_{j} R_{\imath k}=0
$$

where $R_{\imath}$, means the component of the Ricci tensor, i. e. $R_{j k}=R_{j i k}^{\imath}$. If the Ricci tensor is parallel, the curvature is harmonic. However the converse is generally not true, [2]. Concerning this matter, we obtain some results in the case of hypersurfaces in a space of non-negative constant curvature. The purpose of this note is to prove the next theorems:

We denote the $k$-dimensional Euclidean space and the $k$-dimensional sphere of curvature $c$ by $E^{k}$ and $S^{k}(c)$ respectively.

THEOREM 1. Let $M^{n}$ be a connected hypersurface with harmonc curvature, isometrically immersed in $E^{n+1}$ by an isometric immersion $\phi$ with constant mean curvature. We denote the second fundamental form by $h$.

(i) If $M^{n}$ is complete and trace $h^{4}$ is constant on $M^{n}$, then $\phi\left(M^{n}\right)$ is of the form $S^{p} \times E^{n-p}, 0 \leqq p \leqq n$.

(ii) If $M^{n}$ is compact, then $\phi\left(M^{n}\right)$ is $S^{n}$.

THEOREM 2. Let $M^{n}$ be a connected hypersurface with harmonic curvature, isometrically immersed in $S^{n+1}(c)$ by an isometric immersion $\phi$ with constant mean curvature. If $M^{n}$ is complete and trace $h^{4}$ is constant on $M^{n}$, or if $M^{n}$ is compact, then $\phi\left(M^{n}\right)$ is of the form $S^{p}(r) \times S^{n-p}(s), 0 \leqq p \leqq n$, where $r=\alpha^{2}+c$, $s=\beta^{2}+c$, and $\alpha$ and $\beta$ satisf $y \alpha \beta+c=0$ and $p \alpha+(n-p) \beta=$ trace $h$.

The author expresses her sincere gratitude to Professor S. Tachibana for reading the manuscript and valuable advice, and Professor Y. Ogawa for his kind guidance in the course of preparing this note.

\section{The proof of theorems}

We consider a hypersurface $M^{n}$ with harmonic curvature, isometrically

Received June 24, 1985 
immersed in an $(n+1)$-dimensional Riemannian space $\tilde{M}^{n+1}(c)$ of constant curvature $c$ by an isometric immersion $\phi: M^{n} \rightarrow \tilde{M}^{n+1}(c)$, and denote the induced metric tensor, the induced connection, the curvature tensor of $M^{n}$ and the second fundamental form by $g, \nabla, R$ and $h$ respectively. We assume that the mean curvature $\operatorname{tr} h=h_{k}{ }^{k}$ is constant. Under these conditions, the following formulae hold:

$$
R_{\imath j k l}=c\left(g_{i k} g_{j l}-g_{i l} g_{j k}\right)+h_{i k} h_{j l}-h_{i l} h_{j k}
$$

(the Gauss equation),

$$
\begin{array}{cl}
\nabla_{i} h_{j k}-\nabla_{j} h_{i k}=0 & \text { (the Codazzi equation), } \\
\nabla_{i} h_{k}{ }^{k}=0 & \text { (mean curvature constant), } \\
\nabla_{i} R_{j k}-\nabla_{j} R_{i k}=0 & \text { (harmonic curvature), }
\end{array}
$$

where the indices $i, j, k, \cdots$ run from 1 to $n$. The formula (4) implies that the scalar curvature is constant, i. e.

$$
\nabla_{i} R_{k}{ }^{k}=0
$$

On the other hand, we get from (1)

$$
R_{j k}=(n-1) \operatorname{cg}_{j k}+h_{l}{ }^{l} h_{j k}-h_{\jmath}{ }^{l} h_{l k} .
$$

For simplification, we shall write $h^{2}{ }_{\imath \jmath}, h^{3}{ }_{\imath \jmath}, \cdots$ instead of $h_{\imath}{ }^{k} h_{k \jmath}, h^{2}{ }^{k}{ }^{k} h_{k \jmath}, \cdots$. And using (3),

$$
\nabla_{i} R_{j k}=h_{l}{ }^{l} \nabla_{i} h_{j k}-\nabla_{i} h^{2}{ }_{j k} .
$$

Hence we know from (2) and (7) that

$$
\nabla_{i} h^{2}{ }_{j k}-\nabla_{j} h^{2}{ }_{i k}=0
$$

is equivalent to (4). It is easy to see

$$
\nabla_{i} h^{2}{ }^{k}=0
$$

from (7), (3) and (5).

First we shall give two formulae about $\left\|\nabla h^{2}\right\|^{2}=\nabla_{i}\left(h_{j}^{l} h_{l k}\right) \cdot \nabla^{i}\left(h^{\jmath m} h_{m}{ }^{k}\right)$, where $\nabla^{i}=g^{i k} \nabla_{k}$.

LEMMA 1.

$$
\left\|\nabla h^{2}\right\|^{2}=\frac{1}{2} \nabla_{\imath} \nabla^{2}\left(\operatorname{tr} h^{4}\right)-n c \operatorname{tr} h^{4}-\operatorname{tr} h \operatorname{tr} h^{5}+c\left(\operatorname{tr} h^{2}\right)^{2}+\left(\operatorname{tr} h^{3}\right)^{2}
$$


Proof.

$$
\begin{aligned}
\left\|\nabla h^{2}\right\|^{2} & =\left(\nabla_{i} h^{2}{ }_{j k}\right)\left(\nabla^{i} h^{2 j k}\right) \\
& =\nabla_{i}\left(h^{2}{ }_{j k} \nabla^{i} h^{2 j k}\right)-h^{2}{ }_{j k} \nabla_{\imath} \nabla^{i} h^{2 j k} \\
& =\frac{1}{2} \nabla_{\imath} \nabla^{i}\left(\operatorname{tr} h^{4}\right)-h^{2}{ }_{j k} \nabla_{\imath} \nabla^{2} h^{2 j k}
\end{aligned}
$$

holds. Using (1), (3), (8), (9) and the Ricci identity, we get

$$
\begin{aligned}
\nabla_{\imath} \nabla^{\imath} h^{2 j k}= & \nabla_{\imath} \nabla^{\jmath} h^{2 i k} \\
= & \nabla^{j} \nabla_{i} h^{2 i k}+R_{i}{ }^{j l}{ }^{2}{ }^{2}{ }^{k}+R_{i}{ }^{j k l} h^{2 \imath}{ }_{l} \\
= & \left\{(n-1) c g^{j l}+h_{\imath}{ }^{2} h^{j l}-h_{\imath}{ }^{l} h^{j i}\right\} h^{2}{ }^{k} \\
& +\left\{c\left(\delta_{i}{ }^{k} g^{j l}-\delta_{i}{ }^{l} g^{j k}\right)+h_{\imath}{ }^{k} h^{j l}-h_{\imath}{ }^{l} h^{j k}\right\} h^{2 \imath}{ }_{l} .
\end{aligned}
$$

From (11) and (12), the formula (10) follows.

LEMMA 2.

(13) $\quad\left\|\nabla h^{2}\right\|^{2}=\frac{1}{3} \nabla_{\imath} \nabla^{2}\left(\operatorname{tr} h^{4}\right)+\frac{4}{3}\left[\operatorname{tr} h^{4}\left(\operatorname{tr} h^{2}-n c\right)+\operatorname{tr} h\left(c \operatorname{tr} h^{3}-\operatorname{tr} h^{5}\right)\right]$.

Proof. We remark that

$$
\nabla_{i} h^{2}{ }_{j k}=2 h_{j}{ }^{m} \nabla_{i} h_{m k}
$$

holds. In fact,

$$
\nabla_{i} h^{2}{ }_{j k}=\left(\nabla_{i} h_{j}{ }^{m}\right) h_{m k}+h_{j}{ }^{m} \nabla_{i} h_{m k},
$$

implies together with (2) and (8) that the second term of the right side of (15) is symmetric with respect to $i, j$ and $k$, from which (14) follows. Hence

$$
\left\|\nabla h^{2}\right\|^{2}=\left(\nabla_{i} h^{2}{ }_{j k}\right)\left(\nabla^{i} h^{2 j k}\right)=4 h^{2 l}{ }_{m}\left(\nabla_{i} h_{l k}\right)\left(\nabla^{i} h^{m k}\right) .
$$

On the other hand, we have

$$
\begin{aligned}
\left\|\nabla h^{2}\right\|^{2} & =2 h_{\jmath}{ }^{l}\left(\nabla_{i} h_{l k}\right)\left(\nabla^{i} h^{2 j k}\right) \\
& =2 \nabla^{i}\left(h^{3 k l} \nabla_{i} h_{l k}\right)-2 h^{2 j k}\left(\nabla^{i} h_{\jmath}{ }^{l}\right)\left(\nabla_{i} h_{l k}\right)-2 h^{3 k l} \nabla_{\imath} \nabla^{i} h_{l k}
\end{aligned}
$$

by (14). The first and second terms of the right side of (17) are reduced to $\frac{1}{2} \nabla_{\imath} \nabla^{i} h^{4}{ }_{k}{ }^{k}$ and $-\frac{1}{2}\left\|\nabla h^{2}\right\|^{2}$ by (16) respectively. Using (1), (2), (3), (9) and the Ricci identity, we get 


$$
\begin{aligned}
\nabla^{i} \nabla_{i} h_{l k}= & \nabla^{i} \nabla_{l} h_{i k} \\
= & \nabla_{l} \nabla^{i} h_{i k}+R^{\imath}{ }_{l \imath}{ }^{m} h_{m k}+R^{\imath}{ }_{l k}{ }^{m} h_{\imath m} \\
= & \left\{(n-1) c \delta_{l}{ }^{m}+h_{\imath}{ }^{\imath} h_{l}{ }^{m}-h^{\imath m} h_{l i}\right\} h_{m k} \\
& \quad+\left\{c\left(\delta^{\imath}{ }_{k} \delta_{l}{ }^{m}-g^{\imath m} g_{l k}\right)+h^{\imath}{ }_{k} h_{l}{ }^{m}-h^{\imath m} h_{l k}\right\} h_{\imath m} .
\end{aligned}
$$

Therefore the third term of (17) can be reduced to $2\left(-n c \operatorname{tr} h^{4}-\operatorname{tr} h \operatorname{tr} h^{5}\right.$ $+c \operatorname{tr} h \operatorname{tr} h^{3}+\operatorname{tr} h^{2} \operatorname{tr} h^{4}$ ). Finally (17) becomes the formula (13).

We eliminate the term of $\operatorname{tr} h \operatorname{tr} h^{5}$ from (10) and (13), and have

$$
\left\|\nabla h^{2}\right\|^{2}=\nabla_{\imath} \nabla^{2}\left(\operatorname{tr} h^{4}\right)+4\left[\left(\operatorname{tr} h^{3}\right)^{2}-\operatorname{tr} h^{2} \operatorname{tr} h^{4}+c\left(\operatorname{tr} h^{2}\right)^{2}-c \operatorname{tr} h \operatorname{tr} h^{3}\right] .
$$

Taking the suitable orthonormal frame, we diagonize $h$ and denote its diagonal components by $\alpha_{1}, \cdots, \alpha_{n}$. Then the formula (19) can be rewritten to

$$
\left\|\nabla h^{2}\right\|^{2}=\Delta\left(\operatorname{tr} h^{4}\right)-2 \sum_{i \neq j} \alpha_{i} \alpha_{j}\left(\alpha_{i} \alpha_{j}+c\right)\left(\alpha_{i}-\alpha_{j}\right)^{2}
$$

where $\Delta$ means $\nabla^{i} \nabla_{\imath}$.

In the case of $c=0$, if trace $h^{4}$ is constant on $M^{n}$, then all the nonzero eigenvalues of $h$ have a constant unique value on $M^{n}$ by (3) and (20). Therefore we can apply K. Nomizu and B. Smyth's argument if $M^{n}$ is complete. Thus theorem $1(i)$ is proved.

If $M^{n}$ is compact, then we obtain the same result by integrating (20) over $M^{n}$. By the compactness of $M^{n}$, theorem 1(ii) is concluded.

In order to consider the case of $c>0$, we recall the following formula appeared in [3] :

$$
\|\nabla h\|^{2}=\frac{1}{2} \Delta\left(\operatorname{tr} h^{2}\right)-\frac{1}{2} \sum_{i \neq j}\left(\alpha_{i}-\alpha_{j}\right)^{2}\left(\alpha_{i} \alpha_{j}+c\right) .
$$

This formula follows from (1), (2), (3) and the Ricci identity, and in our situation, the first term of the right side of (21) vanishes by (9). So we have

$$
\|\nabla h\|^{2}=-\frac{1}{2} \sum_{i \neq j}\left(\alpha_{i}-\alpha_{j}\right)^{2}\left(\alpha_{i} \alpha_{j}+c\right) .
$$

From (20) and (22), we have

$$
\left\|\nabla h^{2}\right\|^{2}+4 c\|\nabla h\|^{2}=\Delta\left(\operatorname{tr} h^{4}\right)-2 \sum_{i \neq j}\left(\alpha_{i} \alpha_{j}+c\right)^{2}\left(\alpha_{i}-\alpha_{j}\right)^{2},
$$

and theorem 2 is proved as in the case of $c=0$.

Acknowledgement. The method in the case of $c>0$ is due to Professor $\mathrm{H}$. Nakagawa's suggestion, and the author thanks him. 


\section{REFERENCES}

[1] J.P. Bourguignon, Les variétés de dimension 4 à signature non nulle dont la courbure est harmonique sont d'Einstein, Invent. math. 63 (1981), 263-286.

[2] A. DeRDziński, A classification of certain compact Riemannian manifolds with harmonic curvature and non-parallel Ricci tensor, Math. Z. 172 (1980), 273-280.

[3] K. Nomizu And B. Smyth, A formula of Simons' type and hypersurfaces with constant mean curvature, J. Diff. Geom. 3 (1969), 367-377. 